\title{
Comparison of acute pneumonia caused by SARS-COV-2 and other respiratory viruses in children: a retrospective multi-center cohort study during COVID-19 outbreak
}

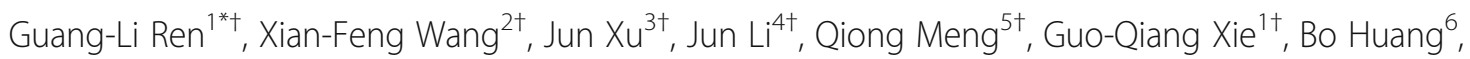
Wei-Chun Zhu' ${ }^{7}$, Jing Lin' ${ }^{7}$, Cheng-He Tang ${ }^{8}$, Sheng Ye ${ }^{9}$, Zhuo Li ${ }^{10}$, Jie Zhu ${ }^{1}$, Zhen Tang ${ }^{1}$, Ming-Xin Ma ${ }^{1}$, Cong Xie ${ }^{11}$, Ying-Wen Wu ${ }^{12}$, Chen-Xi Liu ${ }^{12}$, Fang Yang ${ }^{13}$, Yu-Zong Zhou ${ }^{14}$, Ying Zheng ${ }^{15}$, Shu-Ling Lan ${ }^{16}$, Jian-Feng Chen ${ }^{17}$, Feng $\mathrm{Ye}^{18}$, Yu He${ }^{19}$, Ben-Qing Wu ${ }^{15}$, Long Chen ${ }^{19}$, Si-Mao Fu ${ }^{20^{*}}$, Cheng-Zhong Zheng ${ }^{21^{*}}$ and Yuan Shi ${ }^{1{ }^{*}}$

\footnotetext{
Abstract

Background: Until January 18, 2021, coronavirus disease-2019 (COVID-19) has infected more than 93 million individuals and has caused a certain degree of panic. Viral pneumonia caused by common viruses such as respiratory syncytial virus, rhinovirus, human metapneumovirus, human bocavirus, and parainfluenza viruses have been more common in children. However, the incidence of COVID-19 in children was significantly lower than that in adults. The purpose of this study was to describe the clinical manifestations, treatment and outcomes of COVID19 in children compared with those of other sources of viral pneumonia diagnosed during the COVID-19 outbreak. (Continued on next page)
}

\footnotetext{
*Correspondence: guangliren@hotmail.com; z55319753@163.com; zcz3066@126.com; petshi530@vip.163.com

${ }^{\dagger}$ Guang-Li Ren, Xian-Feng Wang, Jun Xu, Jun Li, Qiong Meng and Guo-Qiang Xie contributed equally to this work.

${ }^{1}$ Department of Pediatrics, General Hospital of Southern Theater Command of PLA, 111 Liuhua Road, Yuexiu District, Guangzhou 510010, Guangdong, China

${ }^{20}$ Department of Pediatrics, Zhongshan Boai Hospital, Zhongshan 528403, Guangdong, China

${ }^{21}$ Department of Pediatrics, Strategic Support Force Medical Center of PLA, Beijing 100101, China

${ }^{19}$ Department of Neonatology, Children's Hospital of Chongqing Medical University/Ministry of Education Key Laboratory of Child/Development and Disorders/National Clinical Research Center for Child Health and Disorders/ Chongqing Key Laboratory of Pediatrics, Chongqing 400014, China Full list of author information is available at the end of the article
}

(c) The Author(s). 2021 Open Access This article is licensed under a Creative Commons Attribution 4.0 International License, which permits use, sharing, adaptation, distribution and reproduction in any medium or format, as long as you give appropriate credit to the original author(s) and the source, provide a link to the Creative Commons licence, and indicate if changes were made. The images or other third party material in this article are included in the article's Creative Commons. licence, unless indicated otherwise in a credit line to the material. If material is not included in the article's Creative Commons licence and your intended use is not permitted by statutory regulation or exceeds the permitted use, you will need to obtain permission directly from the copyright holder. To view a copy of this licence, visit http://creativecommons.org/licenses/by/4.0/ The Creative Commons Public Domain Dedication waiver (http://creativecommons.org/publicdomain/zero/1.0/) applies to the data made available in this article, unless otherwise stated in a credit line to the data. 
(Continued from previous page)

Methods: Children with COVID-19 and viral pneumonia admitted to 20 hospitals were enrolled in this retrospective multi-center cohort study. A total of 64 children with COVID-19 were defined as the COVID-19 cohort, of which 40 children who developed pneumonia were defined as the COVID-19 pneumonia cohort. Another 284 children with pneumonia caused by other viruses were defined as the viral pneumonia cohort. The epidemiologic, clinical, and laboratory findings were compared by Kolmogorov-Smirnov test, t-test, Mann-Whitney $U$ test and Contingency table method. Drug usage, immunotherapy, blood transfusion, and need for oxygen support were collected as the treatment indexes. Mortality, intensive care needs and symptomatic duration were collected as the outcome indicators.

Results: Compared with the viral pneumonia cohort, children in the COVID-19 cohort were mostly exposed to family members confirmed to have COVID-19 (53/64 vs. 23/284), were of older median age (6.3 vs. 3.2 years), and had a higher proportion of ground-glass opacity (GGO) on computed tomography $(18 / 40$ vs. $0 / 38, P<0.001)$. Children in the COVID-19 pneumonia cohort had a lower proportion of severe cases (1/40 vs. 38/284, $P=0.048)$, and lower cases with high fever (3/40 vs. 167/284, $P<0.001)$, requiring intensive care $(1 / 40$ vs. $32 / 284, P<0.047)$ and with shorter symptomatic duration (median 5 vs. $8 \mathrm{~d}, P<0.001$ ). The proportion of cases with evaluated inflammatory indicators, biochemical indicators related to organ or tissue damage, D-dimer and secondary bacterial infection were lower in the COVID-19 pneumonia cohort than those in the viral pneumonia cohort $(P<0.05)$. No statistical differences were found in the duration of positive PCR results from pharyngeal swabs in 25 children with COVID-19 who received antiviral drugs (lopinavir-ritonavir, ribavirin, and arbidol) as compared with duration in 39 children without antiviral therapy [median 10 vs. 9 d, $P=0.885$ ].

Conclusion: The symptoms and severity of COVID-19 pneumonia in children were no more severe than those in children with other viral pneumonia. Lopinavir-ritonavir, ribavirin and arbidol do not shorten the duration of positive PCR results from pharyngeal swabs in children with COVID-19. During the COVID-19 outbreak, attention also must be given to children with infection by other pathogens infection.

Keywords: Children, Severe acute respiratory syndrome, COVID-19, Viral pneumonia

\section{Background}

Coronaviruses are non-segmented positive-stranded RNA viruses, with protrusions on the surface resembling a corona, and with a roughly $30 \mathrm{~kb}$ genome surrounded by a protein envelope [1]. Three coronavirus disease outbreaks have happened in the past two decades: severe acute respiratory syndrome (SARS) [2] in 2003, Middle East respiratory syndrome (MERS) [3] in 2012 and now the Coronavirus Disease 2019 (COVID-19) [4]. Since the first case of COVID-19 was reported in Wuhan, China, in December 2019 until January 18, 2021, SARS-CoV-2 has infected more than 93 million individuals worldwide [5].

Generally, people from all age groups are susceptible to SARS-CoV-2. However, according to data published by the Chinese Center for Disease Control and Prevention (CCDC), the proportion of SARS-CoV-2 infection in children appears to be lower than that of adults [6]. Several studies have described the epidemiologic characteristics and clinical features of children with COVID19, and showed that most children cases had a clear exposure history and the symptoms were milder than adult infection cases [7-9].

Until now, few studies have focused on the treatment of children with COVID-19 and how to identify serious cases during treatment. The similarities and differences between COVID-19 and other sources of viral pneumonia in children, in terms of disease onset, clinical manifestations, test characteristics, and treatment responses, remain to be elucidated. Therefore, we conducted a retrospective multi-center cohort study of acute viral pneumonia in children during the COVID-19 outbreak with the purpose of answering the following three questions: What are the clinical and epidemiological features of children with COVID-19? What do laboratory tests and imaging findings tell us about children with COVID-19 pneumonia as compared with viral pneumonia caused by other viruses? And, what are the differences in treatment experience and outcomes of COVID-19 as compared with those of other viral pneumonia in children?

\section{Methods}

\section{Study design and participants}

This is a retrospective multi-center cohort study. The study protocol was reviewed and approved by the Medical Ethics Committee of General Hospital of Southern Theater Command of Chinese People's Liberation Army (approval number 2020-04). Signed informed consent of participants or their guardians was waived. Children with COVID-19 and pneumonia caused by other viruses diagnosed from December 15, 2019 to March 15, 2020 
during COVID-19 outbreak from 20 hospitals in China were enrolled.

Diagnosis of COVID-19 pneumonia was based on guidelines issued by the National Health Commission of China [10]. Cases with positive results on quantitative RT-PCR tests for SARS-CoV-2 sampled by nasopharyngeal or throat swabs were enrolled into the COVID-19 cohort, of which 40 cases that developed pneumonia were defined as the COVID-19 pneumonia cohort.

The children with pneumonia caused by other viruses were enrolled into the viral pneumonia cohort. The inclusion criteria for the viral pneumonia cohort were: 1 ) Diagnosed with community acquired pneumonia (CAP) based on the guidelines for diagnosis and treatment of CAP in children (2019 version) [11]; 2) Laboratory virus testing confirmed existence of viruses associated with pneumonia, including virus or viral antigen in upperrespiratory specimens (e.g., nasopharyngeal aspirates) and lower respiratory samples (e.g., induced sputum) by culture, immunofluorescence microscopy or molecular diagnostic assays (such as PCR, RT-PCR), and on measurement of antibodies in serum samples; 3) Clinicallydefined pneumonia mainly caused by the detected virus. The exclusion criteria were: 1) Patients older than 18 years old or younger than 28 days; 2) The initial pathogens of pneumonia were mycoplasma pneumonia (MP), bacterial pneumonia or others, and the detected viruses were considered as secondary infection.

\section{Data collection}

Clinical data on epidemiology, signs and symptoms, underlying diseases, laboratory findings, disease diagnosis, and treatment records were retrospectively reviewed by physicians of the 20 hospitals. Data were recorded in a customized data collection form and were checked by two study investigators (RGL and XGQ) independently. Two experienced radiologists (at least 5 years of experience in chest CT) of each hospital were invited to review the X-ray and CT images, and make the case descriptions and radiological diagnoses using uniform standards.

Baseline patient data include age, gender, weight, underlying chronic disease and residential address. Clinical manifestations include respiratory symptoms and signs, gastrointestinal symptoms (diarrhea, vomiting, abdominal pain, and bloating), neurological symptoms (consciousness, response to stimulations, and convulsions), and systemic symptoms (fever, fatigue, and muscle aches). Laboratory findings include whole blood cell tests, biochemical laboratory tests (liver enzymes, creatine kinase $(\mathrm{CK})$, creatine kinase-MB (CK-MB), and lactate dehydrogenase (LDH)), coagulation function (prothrombin time (PT), activated partial thromboplastin time (APTT), fibrinogen, and D-dimer), humoral immune function (IgG, IgA, and IgM), cellular immune function $\left(\mathrm{CD}^{+} / \mathrm{CD}^{+} \mathrm{T}\right.$ cells) and infection indicators (C-reactive protein (CRP), procalcitonin (PCT), erythrocyte sedimentation rate (ESR), interleukin-6 (IL-6)). PCT test value greater than $0.25 \mathrm{ng} / \mathrm{ml}$ was considered as elevated level [12]. The real-time reverse transcriptase polymerase chain-reaction (RT-PCR) test of SARS-CoV-2 was done as described previously [13] and was performed using nasopharyngeal swab, nasal swab and/or rectal swab at the local CCDC.

The severity of SARS-CoV-2 infections were classified as asymptomatic infection, mild infection (without pneumonia), moderate infection (with mild pneumonia), severe infection and critical infection, according to the diagnosis and treatment guidelines for COVID-19 (draft version 7) [10] issued by the National Health Commission of China. Also, the same criteria were used to assess the severity of other viral pneumonia (mild and severe pneumonia) in children.

Drug usage (antiviral drugs, interferon alpha, antibiotics, glucocorticoids, etc.), immunotherapy (immunoglobulin, immunomodulator, etc.), blood transfusion, and need for oxygen support were collected as the treatment indexes. Mortality, intensive care needs and symptomatic duration were collected as the outcome indicators.

\section{Statistical analysis}

All statistical analysis was done using IBM SPSS (version 20, IBM Corp, Armonk, NY, USA). The KolmogorovSmirnov test was used to evaluate distribution type. Normally distributed data are expressed as mean \pm standard deviation (mean $\pm \mathrm{SD}$ ) and independent sample $t$-test was used for comparisons between two cohorts. Nonnormally distributed data and ordinal data are expressed as median (inter-quartile range) and Mann-Whitney $U$ test was used to compare the differences between cohorts. Contingency table method was used to check the proportion of counts data. $P$-values less than 0.05 were considered as statistically significant.

\section{Results}

A total of 64 children patients were included in the COVID-19 cohort, among which 33 patients were from Shenzhen, six patients were from Guangzhou, ten patients were from Wuhan, ten patients were from Huangshi, four were from Zunyi and one was from Hangzhou. From December 15 to March 15, 2020, a total of 4335 cases of children with CAP have been diagnosed in the 20 hospitals, of which 626 cases were clinically diagnosed with viral pneumonia. Finally, 284 viral pneumonia cases with confirmed virus infection were included in the viral pneumonia cohort. The flow chart of participants' recruitment is shown in Fig. 1. 


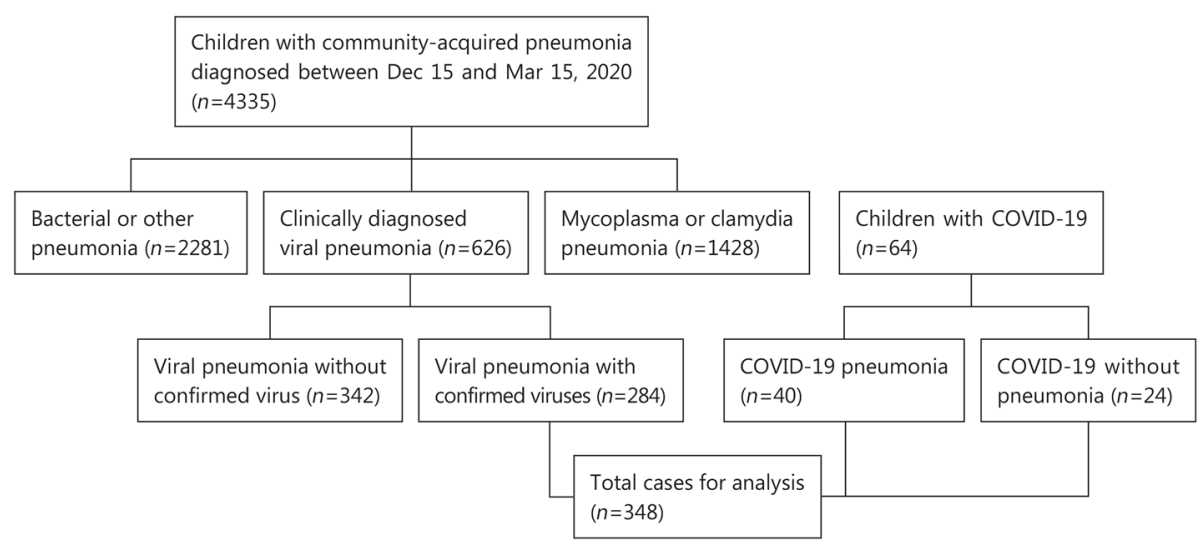

Fig. 1 Flow diagram of recruiting participants. Total of 4335 cases of children CAP had been diagnosed in 20 hospitals, of which 626 cases were clinical diagnosed with viral pneumonia, and finally 284 cases with confirmed other viruses were included, and 64 cases COVID-19 children in the cohort

The epidemiological and baseline characteristics of all 348 participants are shown in Table 1 . Thirty-one children (48.4\%) in the COVID-19 cohort and 161 cases $(56.7 \%)$ in the viral pneumonia cohort were males. No differences were found between two cohorts, while the ages of children in the COVID-19 cohort were older (median 6.3 vs 3.2 years) than those in the viral pneumonia cohort. Two of 64 children in the COVID-19 cohort and 19 of 284 children in the viral pneumonia cohort had underlying chronic diseases. No significant differences were found in baseline characteristics between the two cohorts $(P=0.279)$. Contact with confirmed COVID-infected family members $(53 / 64,82.8 \%)$ was the main exposure mode of COVID-19 children. Household exposure was higher in the COVID-19 cohort than in the viral pneumonia cohort $(53 / 64$ vs. $23 / 284, P<$ 0.001 ). The prevalence of pneumonia in children with COVID-19 was $62.5 \%(40 / 64)$. The ratios of cases progressing from SARS-CoV-2 infection to pneumonia were $4 / 6$ for children younger than 1 year old, $11 / 23$ for $1-5$ years old, $15 / 20$ for $6-10$ years old, and $10 / 15$ for $11-18$ years old. No significant differences were found in the ratios between the different age groups $(P=0.701)$.

To ensure comparability between cohorts, COVID-19 cases with pneumonia were filtered out (COVID-19 pneumonia cohort) to compare with the viral

Table 1 Epidemiological and baseline characteristics of over all 348 participants

\begin{tabular}{|c|c|c|c|}
\hline General data & COVID-19 cohort $(n=64)$ & Viral pneumonia cohort $(n=284)$ & $P$ \\
\hline \multicolumn{4}{|l|}{$\overline{\text { Age }}$} \\
\hline Median (range) & $6.3 \mathrm{yr}$ (3 mon to $18 \mathrm{yr}$ ) & $3.2 \mathrm{yr}(1 \mathrm{mon}$ to $13 \mathrm{yr})$ & $<0.001$ \\
\hline$<1 \mathrm{yr}[n(\%)]$ & $6(9.4)$ & $44(15.5)$ & \multirow[t]{4}{*}{$<0.001$} \\
\hline $1-5 \mathrm{yr}[n(\%)]$ & $23(35.9)$ & $220(77.5)$ & \\
\hline $6-10 \mathrm{yr}[n(\%)]$ & $20(31.2)$ & $19(6.7)$ & \\
\hline $11-18 \mathrm{yr}[n(\%)]$ & $15(18.8)$ & $1(0.4)$ & \\
\hline Male $[n(\%)]$ & $31(48.4)$ & $161(56.7)$ & 0.230 \\
\hline Underlying chronic disease $[n(\%)]$ & $2(3.1)$ & $19(6.7)$ & 0.279 \\
\hline Chronic lung disease (repeated wheezing) & $1(1.6)$ & $14(4.9)$ & 0.231 \\
\hline Heart disease & $0(0.0)$ & $1(0.4)$ & 1.000 \\
\hline Others & $1(1.6)$ & $5(1.8)$ & 1.000 \\
\hline \multicolumn{4}{|l|}{ Exposure history $[n(\%)]^{a}$} \\
\hline Contacted with confirmed family members & $53(82.8)$ & $23(8.1)$ & \multirow[t]{4}{*}{$<0.001$} \\
\hline Contacted with other confirmed cases & $3(4.7)$ & $15(5.3)$ & \\
\hline Travel or residence in epidemic area ${ }^{a}$ & $7(10.9)$ & $0(0.0)$ & \\
\hline No clear exposure history & $1(1.6)$ & $246(86.6)$ & \\
\hline
\end{tabular}

${ }^{a}$ No epidemic area was defined for other respiratory viruses 
pneumonia cohort in terms of clinical manifestations, spectrum of disease severity, and laboratory and imaging findings (Table 2). No significant differences were found in gastrointestinal symptoms (1/40 vs. 17/284) and dry cough (6/40 vs. $35 / 284)$ between the two cohorts, but the proportions of cases with fever $\left(37.3^{\circ} \mathrm{C}, 22 / 40\right.$ vs. $221 / 284)$, high fever $\left(>39.0^{\circ} \mathrm{C}, 3 / 40\right.$ vs. $\left.167 / 284\right)$, cough with sputum production (0/40 vs. $229 / 284)$, rhinitis (1/ 40 vs. $130 / 284)$, and wheezing (0/40 vs. $43 / 284)$ were lower in the COVID-19 pneumonia cohort than in the viral pneumonia cohort $(P<0.05)$. In addition, the duration of fever was shorter in the COVID-19 pneumonia cohort than that in the viral pneumonia cohort [median (IQR), $2(1-3.25) \mathrm{d}$ vs. $4(2-5) \mathrm{d}, P=0.004)]$.

Only one child was in critical condition in the COVID-19 pneumonia cohort, with clinical manifestations including shortness of breath, neurological symptoms (drowsiness), three signs of depression, low blood oxygen saturation and increased PCT, CRP, D-dimer and CK-MB on admission, and had received intubation and invasive ventilator-assisted ventilation immediately after admission.

The proportion of children who developed severe conditions was lower in the COVID-19 pneumonia cohort than in the viral pneumonia cohort (1/40 vs. $38 / 284$, $P=0.048)$. However, no significant differences were found in pneumonia subgroups caused by respiratory syncytial virus (RSV) $(1 / 40$ vs. $19 / 133, P=0.041)$ and human adenovirus $(1 / 40$ vs. $7 / 25, P=0.002)$ as compared to those with pneumonia caused by influenza A and $\mathrm{B}(1 / 40$ vs. $5 / 57, P=0.182)$ or parainfluenza virus $(1 / 40$ vs. $3 / 29, P=0.168)$. The comparison between children with COVID-19 pneumonia and pneumonia caused by the other four common types of respiratory viruses is shown in Table 3.

On admission, counts of white blood cells and lymphocytes of COVID-19 pneumonia children were lower than those of other viral pneumonia $(P<0.05)$. The proportion of cases with leucopenia (white blood cell counts $<5.5 \times 10^{9} / \mathrm{L}$ ) was higher in the COVID-19 cohort (19/40 vs. 38/284). Levels of LDH, ALT, AST, $\mathrm{CK}, \mathrm{D}$-dimer, and the proportion of cases with elevated LDH (13/36 vs. 131/265), CK-MB (5/35 vs. $165 / 270)$, PCT (2/34 vs. $78 / 209)$, CRP (5/38 vs. $80 /$ $281)$, ESR (5/36 vs. $35 / 62)$, IL-6 (5/34 vs. $62 / 168)$ and D-dimer $(>0.5 \mathrm{mg} / \mathrm{L}, 7 / 35$ vs. $18 / 29)$ were lower in the COVID-19 pneumonia cohort as compared with the viral pneumonia cohort $(P<0.05)$. No significant differences were noted between the two cohorts in co-infection with other viruses $(3 / 40$ vs. $22 / 284, P=$ $0.956)$ or co-infection with MP $(9 / 40$ vs. $61 / 284, P=$ 0.883). However, secondary-infection with bacteria was less frequently detected in the COVID-19 pneumonia cohort $(0 / 40$ vs. $52 / 284, P=0.003)$.
Ground-glass opacity (GGO) was the most common radiographic presentation of children with COVID-19 pneumonia. The proportion of cases with GGO was significantly higher $(18 / 40$ vs. $0 / 38, P<0.001)$, while the proportion of cases with consolidation (5/40 vs. $21 / 38$, $P<0.001)$ was lower in the COVID-19 pneumonia cohort as compared to the viral pneumonia cohort. Other radiographic presentations of COVID-19 pneumonia included tiny nodules, consolidation combined with GGO, cable shadow, light shadow, streak shadow and hydrothorax, for which no significant differences were found between COVID-19 pneumonia cohort and the viral pneumonia cohort (Table 2).

Treatments and outcomes of children with COVID-19 pneumonia and other viral pneumonia are listed in Table 4. The proportion of children who received lopinavir-ritonavir, ribavirin and interferon alfa was higher in the COVID-19 pneumonia cohort, but the proportion of children who received oseltamivir was lower, as compared with that in the viral pneumonia cohort $(P<0.05)$. The proportion of children with COVID-19 pneumonia who received antibiotics $(8 / 40$ vs. $261 / 284, P<0.001)$, corticosteroids ( $1 / 40$ vs. $50 / 284, P=0.014)$, immunoglobulin $(1 / 40$ vs. $39 / 284, P=0.043)$ and who needed oxygen support $(P=0.024)$ were lower than that of those with other viral pneumonia.

Evaluation of the effects of three antiviral drugs through comparisons within the COVID-19 cohort showed that lopinavir-ritonavir (lopinavir $6-10 \mathrm{mg} / \mathrm{kg}$ and ritonavir $1.5-2.5 \mathrm{mg} / \mathrm{kg}$ per day, median for 9 days) was used in $12(18.8 \%)$ cases, ribavirin $(10-15 \mathrm{mg} / \mathrm{kg}$ per day, median for 8 days) was used in $8(12.5 \%)$ cases, and arbidol (5-8 $\mathrm{mg} / \mathrm{kg}$ per day, median for 7 days) was used in $6(9.4 \%)$ children with COVID-19. The duration of positive PCR results from pharyngeal swabs was not significantly different between 25 COVID-19 children receiving those antiviral drugs and that of 39 control cases not receiving those antiviral drugs [median (IQR), 10 (5-13.5) d vs 9 (7-11) d, $P=0.885$ ]. Subgroup analysis performed to evaluate the effectiveness of certain antiviral drugs showed that no significant differences were found in the duration of positive PCR results from pharyngeal swabs in children with COVID-19 who used lopinavir-ritonavir, ribavirin, or arbidol with nonantiviral therapy controls $(P<0.05$, Fig. 2$)$.

Up until April 15, 2020, only one patient in the viral pneumonia cohort died and the other 347 patients were discharged, with no significant differences in the mortality rate between the two cohorts. As compared with the viral pneumonia cohorts, the proportion of cases that required intensive care was lower $(1 / 40$ vs. $32 / 284, P=$ $0.047)$ and the symptomatic duration was shorter [median (IQR), $5(0-8)$ d vs. $8(4-12) \mathrm{d}, P<0.001]$ in the COVID-19 pneumonia cohort. 
Table 2 Symptoms, signs, disease severity, laboratory tests and radiographic findings of COVID-19 pneumonia and other viral pneumonia

\begin{tabular}{|c|c|c|c|}
\hline Measures & COVID-19 pneumonia $(n=40)^{a}$ & Viral pneumonia $(n=284)^{a}$ & $P$ \\
\hline \multicolumn{4}{|l|}{ Symptoms and signs [n (\%)] } \\
\hline Fever & $22(55.0)$ & $221(77.8)$ & $<0.001$ \\
\hline Duration of fever [d, median (IQR)] & $2.00(1.00-3.25)$ & $4.00(2.00-5.00)$ & 0.004 \\
\hline \multicolumn{4}{|l|}{ Highest temperature [n (\%)] } \\
\hline$<37.3^{\circ} \mathrm{C}$ & $18(45.0)$ & $63(22.2)$ & $<0.001$ \\
\hline $37.4-37.9^{\circ} \mathrm{C}$ & $8(20.0)$ & $8(2.8)$ & \\
\hline $38.0-38.9^{\circ} \mathrm{C}$ & $11(27.5)$ & $46(16.2)$ & \\
\hline$>39.0^{\circ} \mathrm{C}$ & $3(7.5)$ & $167(58.8)$ & \\
\hline Cough $[n(\%)]$ & $6(15.0)$ & $256(90.1)$ & $<0.001$ \\
\hline Dry cough $[n(\%)]$ & $6(15.0)$ & $35(12.3)$ & 0.634 \\
\hline Cough with Sputum production [n (\%)] & $0(0.0)$ & 229 (80.6) & $<0.001$ \\
\hline Sore throat $[n(\%)]$ & $1(2.5)$ & $8(2.8)$ & 0.909 \\
\hline Rhinitis [n (\%)] & $1(2.5)$ & $130(45.8)$ & $<0.001$ \\
\hline Short of breath $[n(\%)]$ & $1(2.5)$ & $22(7.7)$ & 0.226 \\
\hline Wheezing $[n(\%)]$ & $0(0.0)$ & $43(15.1)$ & 0.017 \\
\hline Gastrointestinal symptoms [n (\%)] & $1(2.5)$ & $17(6.0)$ & 0.709 \\
\hline Fatigue or muscle aches [n (\%)] & $1(2.5)$ & $12(4.2)$ & 0.603 \\
\hline Neurological symptoms [n (\%)] & $2(5.0)$ & $15(5.3)$ & 0.940 \\
\hline Three depression sign [n (\%)] & $1(2.5)$ & $36(12.7)$ & 0.058 \\
\hline Low $\mathrm{SaO}_{2}$ of inhospital $(<95 \%, n(\%)]$ & $1(2.5)$ & $9(3.2)$ & 0.819 \\
\hline \multicolumn{4}{|l|}{ Severity $[n(\%)]$} \\
\hline Moderate & $39(97.5)$ & $246(86.6)$ & 0.048 \\
\hline Server or critical & $1(2.5)$ & $38(13.4)$ & \\
\hline \multicolumn{4}{|l|}{ Blood routine } \\
\hline WBC count $\left[\times 10^{9} / \mathrm{L} ;\right.$ median $\left.(\mathrm{IQR})\right]$ & $5.3(4.4-7.8)$ & $8.6(6.3-12.1)$ & $<0.001$ \\
\hline$<5.5 \times 10^{9} / L[n(\%)]$ & $19(47.5)$ & $38(13.4)$ & $<0.001$ \\
\hline Neutrophil count $\left[\times 10^{9} / \mathrm{L}\right.$; median $\left.(\mathrm{IQR})\right]$ & $2.5(1.9-3.3)$ & $3.2(2.1-4.6)$ & 0.029 \\
\hline$<1.1 \times 109 / L[n(\%)]$ & $3(7.5)$ & $12(4.2)$ & 0.356 \\
\hline Platelet count $\left[\times 10^{9} / \mathrm{L} ;\right.$ median $\left.(\mathrm{IQR})\right]$ & $253.0(217.3-320.0)$ & $275.0(209.0-350.0)$ & 0.410 \\
\hline$<120 \times 10^{9} / L[n(\%)]$ & $1(2.5)$ & $9(3.2)$ & 0.819 \\
\hline \multicolumn{4}{|l|}{ Inflammatory indicators [n (\%)] } \\
\hline $\mathrm{PCT}(>0.25 \mathrm{ng} / \mathrm{ml})$ & 2/34 (5.9) & 78/209 (37.3) & $<0.001$ \\
\hline CRP $(>10 \mathrm{mg} / \mathrm{L})$ & 5/38 (13.2) & 80/281 (28.5) & 0.045 \\
\hline $\operatorname{ESR}(>20 \mathrm{~s})$ & $5 / 36(13.9)$ & 35/62 (56.5) & $<0.001$ \\
\hline IL-6 (> 20.9 ng/L) & $5 / 34(14.7)$ & 62/168 (36.9) & 0.012 \\
\hline \multicolumn{4}{|l|}{ Blood biochemistry } \\
\hline LDH [U/L; median (IQR)] & $210.0(187.0-482.4)$ & $349.0(228.5-418.5)$ & $<0.001$ \\
\hline$>300$ U/L [n (\%)] & $13 / 26(36.1)$ & 131/265 (49.4) & $<0.001$ \\
\hline $\mathrm{ALT}[\mathrm{U} / \mathrm{L} ;$ median (IQR)] & $12.5(9.25-24.0)$ & $17(13-23)$ & 0.035 \\
\hline$>45 \mathrm{U} / \mathrm{L}[n(\%)]$ & $4(10.0)$ & 18/275 (6.5) & 0.432 \\
\hline AST [U/L; median (IQR)] & $33.9(19.8-41.3)$ & $37.0(31.0-45.0)$ & 0.011 \\
\hline$>50 \mathrm{U} / \mathrm{L}[n(\%)]$ & $4(10.0)$ & 36/276 (13.0) & 0.588 \\
\hline CK [U/L; median (IQR)] & $70.0(57.0-91.8)$ & $110.5(79.3-155.8)$ & $<0.001$ \\
\hline
\end{tabular}


Table 2 Symptoms, signs, disease severity, laboratory tests and radiographic findings of COVID-19 pneumonia and other viral pneumonia (Continued)

\begin{tabular}{|c|c|c|c|}
\hline Measures & COVID-19 pneumonia $(n=40)^{a}$ & Viral pneumonia $(n=284)^{a}$ & $P$ \\
\hline$>185 \mathrm{U} / \mathrm{L}[n(\%)]$ & 2/35 (5.7) & 48/276 (17.4) & 0.076 \\
\hline CK-MB (> $27 \mathrm{U} / \mathrm{L})[n(\%)]$ & $5 / 35(14.3)$ & $165 / 270(61.1)$ & $<0.001$ \\
\hline \multicolumn{4}{|l|}{ Humoral immunity [g/L; median (IQR)] } \\
\hline $\lg G$ & $8.1(4.8-10.6)$ & $8.4(6.9-10.2)$ & 0.712 \\
\hline $\lg M$ & $1.3(1.0-1.6)$ & $1.3(0.9-1.6)$ & 0.995 \\
\hline $\lg A$ & $1.0(0.5-1.3)$ & $0.9(0.6-1.4)$ & 0.218 \\
\hline \multicolumn{4}{|l|}{ Cellular immunity } \\
\hline $\mathrm{CD}^{+} / \mathrm{CD}^{+} \mathrm{T}$ cell [median (IQR)] & $1.2(0.9-1.6)$ & $1.2(0.9-1.8)$ & 0.534 \\
\hline$<0.96[n(\%)]$ & 6/17 (35.3) & $4 / 16(25.0)$ & 0.479 \\
\hline $0.96-2.05[n(\%)]$ & 10/17 (58.8) & $9 / 16(56.2)$ & \\
\hline$>2.05[n(\%)]$ & $1 / 17(5.9)$ & 3/16 (18.8) & \\
\hline \multicolumn{4}{|l|}{ Coagulation } \\
\hline Fibrinogen [g/L; median (IQR)] & $2.5(2.4-3.0)$ & $3.1(2.4-3.9)$ & 0.114 \\
\hline D-dimer [mg/L; median (IQR)] & $0.32(0.25-0.42)$ & $0.53(0.33-1.00)$ & 0.004 \\
\hline$>0.5 \mathrm{mg} / \mathrm{L}[n(\%)]$ & $7 / 35(20.0)$ & $18 / 29(62.1)$ & 0.001 \\
\hline Prothrombin time [s, median (IQR)] & $11.2(10.2-13.3)$ & $11.2(10.3-13.0)$ & 0.113 \\
\hline APTT $[s$, median $(\mathrm{IQR})]$ & $34.7(31.3-36.4)$ & $31.6(25.8-35.7)$ & 0.111 \\
\hline \multicolumn{4}{|l|}{ Co-infection $[n(\%)]$} \\
\hline Virus & $3(7.5)$ & $22(7.7)$ & 0.956 \\
\hline MP & $9(22.5)$ & $61(21.5)$ & 0.883 \\
\hline Secondary-infection with bacteria $[n(\%)]$ & $0(0.0)$ & $52(18.3)$ & 0.003 \\
\hline \multicolumn{4}{|c|}{ Affected area on radiography $[n(\%)] —$ no. (\%) } \\
\hline Left lung lobe & $5(12.5)$ & 18/202 (8.9) & 0.071 \\
\hline Right lung lobe & $14(35.0)$ & $41 / 202(20.3)$ & \\
\hline Bilateral lung lobe & $21(52.5)$ & 143/202 (70.8) & \\
\hline \multicolumn{4}{|l|}{$\mathrm{CT}$ images of the chest $[n(\%)]$} \\
\hline GGO & $18(45.0)$ & $0 / 38(0.0)$ & $<0.001$ \\
\hline Tiny nodules & $6(15.0)$ & 2/38 (5.3) & 0.297 \\
\hline Consolidation & $5(12.5)$ & 21/38 (55.3) & $<0.001$ \\
\hline Consolidation combined with GGO & $4(10.0)$ & $1 / 38(2.6)$ & 0.387 \\
\hline Cable shadow & $11(27.5)$ & 18/38 (47.4) & 0.070 \\
\hline Light shadow & $6(15.0)$ & 5/38 (13.2) & 0.815 \\
\hline Streak shadow & $6(15.0)$ & 6/38 (15.8) & 0.923 \\
\hline Hydrothorax & $1(2.5)$ & $1 / 38(2.6)$ & 1.000 \\
\hline
\end{tabular}

The denominator of the proportional count in the table was the total number of evaluated cases, and the numerator is the number of cases positive on this index $W B C$ White blood cell, PCT Procalcitonin, CRP C-reactive protein, ESR Erythrocyte sedimentation rate, IL- 6 Interleukin-6, $L D H$ Lactate dehydrogenase, $A L T$ Alanine aminotransferase, AST Aspartate aminotransferase, CK Creatine kinase, CK-MB Creatine kinase - MB, APTT Activated partial thromboplastin time, MP Mycoplasma pneumonia, GGO Ground-glass opacity

${ }^{a}$ Missing data are present by a fraction

\section{Discussion}

Results of this multi-center retrospective cohort study provided new insight into the clinical features and treatment of children with COVID-19. Comparison of the data of 64 children with COVID-19 with the data of 284 children patients with other types of viral pneumonia identified differences in clinical manifestations, laboratory parameters, imaging characteristics and treatment. COVID-19 children had a lower proportion of severe cases, lower cases with high fever, cases requiring intensive care and overall shorter symptomatic duration. Cases with inflammatory indicators, biochemical 
Table 3 The comparison between pneumonia caused by SARS-COV-2 and other four common kinds of respiratory viruses ${ }^{*}$

\begin{tabular}{|c|c|c|c|c|c|}
\hline Measures & $\begin{array}{l}\text { COVID-19 } \\
\text { pneumonia } \\
(n=40)\end{array}$ & $\begin{array}{l}\text { RSV } \\
\text { pneumonia } \\
(n=133)\end{array}$ & $\begin{array}{l}\text { Influenza A and B } \\
\text { pneumonia }(n=57)\end{array}$ & $\begin{array}{l}\text { Parainfluenza virus } \\
\text { pneumonia }(n=29)\end{array}$ & $\begin{array}{l}\text { Human adenovirus } \\
\text { pneumonia }(n=25)\end{array}$ \\
\hline \multicolumn{6}{|l|}{ Age } \\
\hline Median (yr) & 7.0 & 2.6 & 4.0 & 3.4 & 3.9 \\
\hline$<1 \mathrm{yr}[n(\%)]$ & $4(10.0)$ & $25(18.8)$ & $6(10.5)$ & $5(17.2)$ & $1(4.0)$ \\
\hline $1-5 \mathrm{yr}[n(\%)]$ & $11(27.5)$ & $108(81.2)$ & $42(73.7)$ & $23(79.3)$ & $17(68.0)$ \\
\hline $6-10 y r[n(\%)]$ & $15(37.5)$ & $0(0.0)$ & $8(14.0)$ & $1(3.4)$ & $7(28.0)$ \\
\hline $11-18 y r[n(\%)]$ & $10(25.0)$ & $0(0.0)$ & $1(1.8)$ & $0(0.0)$ & $0(0.0)$ \\
\hline Male $[n(\%)]$ & $18(45.0)$ & $73(54.9)$ & $30(52.6)$ & $19(65.5)$ & $16(64.0)$ \\
\hline $\begin{array}{l}\text { Underlying chronic } \\
\text { disease }[n(\%)]\end{array}$ & $2(5.0)$ & $10(7.5)$ & $3(5.3)$ & $1(3.4)$ & $2(8.0)$ \\
\hline $\begin{array}{l}\text { Chronic lung disease } \\
\text { (repeated wheezing) }\end{array}$ & $1(2.5)$ & $5(3.8)$ & $2(3.5)$ & $1(3.4)$ & $2(8.0)$ \\
\hline Heart disease & $0(0.0)$ & $1(0.8)$ & $0(0.0)$ & $0(0.0)$ & $0(0.0)$ \\
\hline Others & $1(2.5)$ & $4(3.0)$ & $1(1.8)$ & $0(0.0)$ & $0(0.0)$ \\
\hline \multicolumn{6}{|l|}{ Symptoms and signs } \\
\hline Fever $[n(\%)]$ & $22(55.0)$ & $105(78.9)$ & $49(86.0)$ & $15(51.7)$ & $22(88.0)$ \\
\hline $\begin{array}{l}\text { Duration of fever [d, } \\
\text { median (IQR)] }\end{array}$ & $2(1-3)$ & $3(2-5)$ & $4(2.5-6.0)$ & $3(2-4)$ & $5(2.00-8.25)$ \\
\hline \multicolumn{6}{|c|}{ Highest temperature $[n(\%)]$} \\
\hline$<37.3^{\circ} \mathrm{C}$ & $18(45.0)$ & $28(21.0)$ & $8(14.0)$ & $14(48.3)$ & $3(12.0)$ \\
\hline $37.4-37.9^{\circ} \mathrm{C}$ & $8(20.0)$ & $7(5.3)$ & $0(0.0)$ & $0(0.0)$ & $0(0.0)$ \\
\hline $38.0-38.9^{\circ} \mathrm{C}$ & $11(27.5)$ & $23(17.3)$ & $12(21.0)$ & $4(13.8)$ & $0(0.0)$ \\
\hline$>39.0^{\circ} \mathrm{C}$ & $3(7.5)$ & $75(56.4)$ & $37(64.9)$ & $11(37.9)$ & $22(88.0)$ \\
\hline Cough $[n(\%)]$ & $6(15.0)$ & $119(89.5)$ & $53(93.0)$ & $26(89.6)$ & $19(76.0)$ \\
\hline Dry cough [n (\%)] & $6(15.0)$ & $12(9.0)$ & $8(14.0)$ & $1(3.4)$ & $6(24.0)$ \\
\hline $\begin{array}{l}\text { Cough with sputum } \\
\text { production }[n(\%)]\end{array}$ & $0(0.0)$ & $119(89.5)$ & $45(78.9)$ & $25(86.2)$ & $13(52.0)$ \\
\hline Sore throat $[n(\%)]$ & $1(2.5)$ & $2(1.5)$ & $5(8.8)$ & $0(0.0)$ & $1(4.0)$ \\
\hline Rhinitis [n (\%)] & $1(2.5)$ & $65(48.9)$ & $21(36.8)$ & $18(62.1)$ & $8(32.0)$ \\
\hline Short of breath $[n(\%)]$ & $1(2.5)$ & $13(9.8)$ & $3(5.3)$ & $1(3.4)$ & $2(8.0)$ \\
\hline Wheezing $[n(\%)]$ & $0(0.0)$ & $28(21.0)$ & $3(5.3)$ & $2(6.9)$ & $2(8.0)$ \\
\hline $\begin{array}{l}\text { Gastrointestinal } \\
\text { symptoms [n (\%)] }\end{array}$ & $1(2.5)$ & $7(5.3)$ & $4(7.0)$ & $2(6.9)$ & $1(4.0)$ \\
\hline $\begin{array}{l}\text { Fatigue or muscle aches } \\
{[n(\%)]}\end{array}$ & $1(2.5)$ & $2(1.5)$ & $10(17.5)$ & $0(0.0)$ & $0(0.0)$ \\
\hline $\begin{array}{l}\text { Neurological symptoms } \\
{[n(\%)]}\end{array}$ & $2(5.0)$ & $8(6.0)$ & $6(10.5)$ & $0(0.0)$ & $0(0.0)$ \\
\hline $\begin{array}{l}\text { Three depressions sign } \\
{[n(\%)]}\end{array}$ & $1(2.5)$ & $20(15.0)$ & $3(5.3)$ & $3(10.3)$ & $5(20.0)$ \\
\hline $\begin{array}{l}\text { Low } \mathrm{SaO}_{2} \text { of inhospital } \\
(\leq 95 \%)[n(\%)]\end{array}$ & $1(2.5)$ & $4(3.0)$ & $1(1.8)$ & $1(3.4)$ & $3(12.0)$ \\
\hline $\begin{array}{l}\text { Symptom duration [D; } \\
\text { median (IQR)] }\end{array}$ & $5(0-8)$ & $8(5-10)$ & $8(5-10)$ & $9(6-11)$ & $9(4-12)$ \\
\hline \multicolumn{6}{|l|}{ Severity $[n(\%)]$} \\
\hline Moderate & $39(97.5)$ & $114(85.7)$ & $52(91.2)$ & $26(89.7)$ & $18(72.0)$ \\
\hline Server or critical & $1(2.5)$ & 19 (14.3) & $5(8.8)$ & $3(10.3)$ & $7(28.0)$ \\
\hline
\end{tabular}

Blood routine 
Table 3 The comparison between pneumonia caused by SARS-COV-2 and other four common kinds of respiratory viruses ${ }^{*}$ (Continued)

\begin{tabular}{|c|c|c|c|c|c|}
\hline Measures & $\begin{array}{l}\text { COVID-19 } \\
\text { pneumonia } \\
(n=40)\end{array}$ & $\begin{array}{l}\text { RSV } \\
\text { pneumonia } \\
(n=133)\end{array}$ & $\begin{array}{l}\text { Influenza A and B } \\
\text { pneumonia }(n=57)\end{array}$ & $\begin{array}{l}\text { Parainfluenza virus } \\
\text { pneumonia }(n=29)\end{array}$ & $\begin{array}{l}\text { Human adenovirus } \\
\text { pneumonia }(n=25)\end{array}$ \\
\hline $\begin{array}{l}\text { WBC }\left[\times 10^{9} / L ; \text { median }\right. \\
(\text { IQR) }]\end{array}$ & $5.3(4.4-7.8)$ & $8.6(6.3-11.5)$ & $7.0(5.7-9.8)$ & $7.7(6.0-12.3)$ & $9.3(6.9-14.3)$ \\
\hline$<5.5 \times 10^{9} / \mathrm{L}[n(\%)]$ & $19(47.5)$ & $17(12.8)$ & $14(24.6)$ & $4(13.8)$ & $2(8.0)$ \\
\hline $\begin{array}{l}\text { Neutrophil count }\left[\times 10^{9} /\right. \\
\text { L,median (IQR) }]\end{array}$ & $2.5(1.9-3.3)$ & $3.5(2.2-4.8)$ & $2.3(1.6-3.9)$ & $3.3(2.6-4.9)$ & $3.0(1.8-4.4)$ \\
\hline$<1.1 \times 10^{9} / \mathrm{L}[n(\%)]$ & $3(7.5)$ & $0(0.0)$ & $10(17.5)$ & $0(0.0)$ & $1(4.0)$ \\
\hline $\begin{array}{l}\text { Platelet count }\left[\times 10^{9} / \mathrm{L} \text {, }\right. \\
\text { median }(\mathrm{IQR})]\end{array}$ & $\begin{array}{l}253.0(217.2 .0- \\
320.0)\end{array}$ & $\begin{array}{l}285.0(224.0- \\
366.8)\end{array}$ & $246.5(187.3-298.5)$ & $262.0(173.5-325.0)$ & $259.0(193.0-313.8)$ \\
\hline$<120 \times 10^{9} / \mathrm{L}[n(\%)]$ & $1(2.5)$ & $5(3.8)$ & $2(3.5)$ & $1(3.4)$ & $1(4.0)$ \\
\hline \multicolumn{6}{|l|}{ Infection biomarkers [n (\%)] } \\
\hline $\mathrm{PCT}(>0.25 \mathrm{ng} / \mathrm{ml})$ & 2/34 (5.9) & 28/88 (31.8) & 15/46 (32.6) & 7/19 (36.8) & $14 / 21(66.7)$ \\
\hline CRP $(>10 \mathrm{mg} / \mathrm{L})$ & 5/38 (13.2) & 25/131 (19.1) & $24(42.1)$ & $4 / 28(14.3)$ & $16(64.0)$ \\
\hline ESR (> $20 s)$ & 5/36 (13.9) & $12 / 22(54.5)$ & 10/18 (55.6) & $1 / 5(20.0)$ & $9 / 12(7.5)$ \\
\hline IL-6 (> 20.9 ng/L) & 5/34 (14.7) & 25/80 (31.2) & 9/31 (29.0) & 6/17 (35.3) & $15 / 23(65.2)$ \\
\hline \multicolumn{6}{|l|}{ Blood biochemistry } \\
\hline LDH [U/L, median (IQR)] & $210.0(187.0-482.4)$ & $\begin{array}{l}361.0(303.5- \\
418.5)\end{array}$ & 363.5 (269.5-489.8) & 310.5 (288.0-361.8) & $342.0(286.0-405.0)$ \\
\hline$>300$ U/L [n (\%)] & $13 / 36(36.1)$ & 97/125 (77.6) & $33 / 50(66.0)$ & 18/28 (64.3) & $17(68.0)$ \\
\hline $\mathrm{ALT}[\mathrm{U} / \mathrm{L}$, median (IQR)] & $12.5(9.25-24.0)$ & $18.0(14.0-24.0)$ & $15.0(12.3-20.8)$ & 16.5 (13.0-19.0) & $15.5(9.3-19.3)$ \\
\hline$>45 \mathrm{U} / \mathrm{L}[n(\%)]$ & $4(10.0)$ & $7 / 130(5.4)$ & $3 / 56(5.4)$ & $0 / 28(0.0)$ & $5 / 24(20.8)$ \\
\hline AST [U/L, median (IQR)] & $33.9(19.8-41.3)$ & $38.5(33.0-46.0)$ & $39.0(28.5-46.8)$ & $36.5(32.3-42.3)$ & $29.0(23.0-37.5)$ \\
\hline$>50 \mathrm{U} / \mathrm{L}[n(\%)]$ & $4(10.0)$ & 19/130 (14.6) & $8 / 56(14.3)$ & $2 / 28(7.1)$ & $1(4.0)$ \\
\hline CK [U/L, median (IQR)] & $70.0(57.0-91.8)$ & $\begin{array}{l}113.0(82.0- \\
154.3)\end{array}$ & $122.5(88.0-179.0)$ & $110.5(84.3-181.0)$ & $86.0(49.0-154.5)$ \\
\hline$>185 \mathrm{U} / \mathrm{L}[n(\%)]$ & 2/35 (5.7) & 23/130 (17.7) & $12 / 56(21.4)$ & $7 / 28(25.0)$ & $4(16.0)$ \\
\hline CK-MB (> $27 \mathrm{U} / \mathrm{L})[n(\%)]$ & $5 / 35(14.3)$ & $87 / 126(69.0)$ & $21 / 56(37.5)$ & $22(75.9)$ & $15 / 24(62.5)$ \\
\hline \multicolumn{6}{|c|}{ Humoral immunity [g/L, median (IQR)] } \\
\hline $\lg G$ & $8.1(4.8-10.6)$ & $8.4(6.4-10.2)$ & $8.4(6.9-10.6)$ & $8.5(6.5-10.6)$ & $8.9(7.2-9.9)$ \\
\hline $\lg M$ & $1.3(1.0-1.6)$ & $1.3(0.8-1.8)$ & $1.2(1.1-1.5)$ & $1.3(1.0-1.8)$ & $1.3(0.9-1.5)$ \\
\hline $\lg A$ & $1.0(0.5-1.3)$ & $0.8(0.5-0.1 .3)$ & $0.9(0.5-1.4)$ & $0.9(0.6-1.4)$ & $1.0(0.7-1.2)$ \\
\hline \multicolumn{6}{|l|}{ Co-infection $[n(\%)]$} \\
\hline Virus $^{*}$ & $3(7.5)$ & & & & \\
\hline $\begin{array}{l}\text { Mycoplasma } \\
\text { pneumoniae }\end{array}$ & $9(22.5)$ & $21(15.8)$ & $16(28.1)$ & $2(6.9)$ & $9(36.0)$ \\
\hline $\begin{array}{l}\text { Secondary-infection with } \\
\text { Bacteria }[n(\%)]\end{array}$ & $0(0.0)$ & $25(18.8)$ & $6(10.5)$ & $6(20.7)$ & $5(20.0)$ \\
\hline \multicolumn{6}{|c|}{$\begin{array}{l}\text { Affected area on radiography }[n(\%)] \\
- \text { no. (\%) }\end{array}$} \\
\hline Left lung lobe & $5(12.5)$ & $2 / 81(2.5)$ & 9/52 (17.3) & 2/23 (8.7) & $0 / 17(0.0)$ \\
\hline Right lung lobe & $14(35.0)$ & 16/81 (19.7) & $17 / 52(32.7)$ & 3/23 (13.0) & $1 / 17(5.9)$ \\
\hline Bilateral lung lobe & $21(52.5)$ & 63/81 (77.8) & $26 / 52(50.0)$ & 18/23 (78.3) & $16 / 17(94.1)$ \\
\hline \multicolumn{6}{|l|}{$C T$ images of the chest $[n(\%)]$} \\
\hline GGO & $18(45.0)$ & $0 / 12(0.0)$ & $0 / 9(0.0)$ & $0 / 2(0.0)$ & $0 / 7(0.0)$ \\
\hline Tiny nodules & $6(15.0)$ & $0 / 12(0.0)$ & $1 / 9(11.1)$ & $0 / 2(0.0)$ & $0 / 7(0.0)$ \\
\hline Consolidation & $5(12.5)$ & $2 / 12(16.7)$ & 8/9 (88.9) & $1 / 2(50.0)$ & 4/7 (57.1) \\
\hline
\end{tabular}


Table 3 The comparison between pneumonia caused by SARS-COV-2 and other four common kinds of respiratory viruses ${ }^{*}$ (Continued)

\begin{tabular}{llllll}
\hline Measures & $\begin{array}{l}\text { COVID-19 } \\
\text { pneumonia } \\
(\boldsymbol{n}=\mathbf{4 0})\end{array}$ & $\begin{array}{l}\text { RSV } \\
\text { pneumonia } \\
(\boldsymbol{n}=133)\end{array}$ & $\begin{array}{l}\text { Influenza A and B } \\
\text { pneumonia }(\boldsymbol{n}=\mathbf{5 7})\end{array}$ & $\begin{array}{l}\text { Parainfluenza virus } \\
\text { pneumonia }(\boldsymbol{n}=\mathbf{2 9})\end{array}$ & $\begin{array}{l}\text { Human adenovirus } \\
\text { pneumonia }(\boldsymbol{n}=\mathbf{2 5})\end{array}$ \\
\hline $\begin{array}{l}\text { Consolidation combined } \\
\text { with GGO }\end{array}$ & $4(10.0)$ & $1 / 12(8.3)$ & $0 / 9(0.0)$ & $0 / 2(0.0)$ & $0 / 7(0.0)$ \\
Cable shadow & $11(27.5)$ & $5 / 12(41.7)$ & $4 / 9(44.4)$ & $0 / 2(0.0)$ & $5 / 7(71.4)$ \\
Light shadow & $6(15.0)$ & $1 / 12(8.3)$ & $2 / 9(22.2)$ & $1 / 2(50.0)$ & $0 / 7(0.0)$ \\
Streak shadow & $6(15.0)$ & $5 / 12(41.7)$ & $0 / 9(0.0)$ & $0 / 2(0.0)$ & $0 / 7(0.0)$ \\
Hydrothorax & $1(2.5)$ & $0 / 12(0.0)$ & $0 / 9(0.0)$ & $0 / 2(0.0)$ & $1 / 7(14.3)$ \\
\hline
\end{tabular}

*. Missing data are present by a fraction. WBC. White blood cell. PCT. Procalcitonin. CRP. C-reactive protein. ESR. Erythrocyte sedimentation rate. IL-6. Interleukin-6. LDH. Lactate dehydrogenase. ALT. Alanine aminotransferase. AST. Aspartate aminotransferase. CK. Creatine kinase. CK-MB. Creatine kinase - MB. RSV. respiratory syncytial virus. GGO. Ground-glass opacity. *The blank in the column of co-infection was because viral pneumonia caused by multiple viruses was not included in this comparison. Of the 284 cases of viral pneumonia, 133 were caused by RSV, 57 were caused by influenza A or influenza B, 29 were caused by parainfluenza virus, 25 were caused by human adenovirus, 22 were caused by multiple viruses, six were caused by human rhinovirus, six were caused by human Boca virus and six cases were caused by other viruses. The comparison was made between pneumonia caused by SARS-COV-2 and other four common kinds of respiratory viruses

Table 4 Treatments and outcome of COVID-19 pneumonia and other viral pneumonia

\begin{tabular}{|c|c|c|c|}
\hline Measures & COVID-19 pneumonia $(n=40)$ & Viral pneumonia $(n=284)$ & $P$ \\
\hline Antiviral therapy $[$ Yes, $n(\%)]$ & $14(35.0)$ & $71(25.0)$ & 0.178 \\
\hline Lopinavir-ritonavir & $9(22.5)$ & $0(0.0)$ & $<0.001$ \\
\hline Ribavirin & $4(10.0)$ & $2(0.7)$ & $<0.001$ \\
\hline Arbidol & $1(2.5)$ & $0(0.0)$ & 0.252 \\
\hline Oseltamivir & $1(2.5)$ & $65(22.9)$ & 0.003 \\
\hline Paramive & $0(0.0)$ & $4(1.4)$ & 1.000 \\
\hline Others & $0(0.0)$ & $3(1.1)$ & 1.000 \\
\hline Interferon alfa [n (\%)] & $38(95.0)$ & $15(5.3)$ & $<0.001$ \\
\hline Antibiotic therapy [Yes, $n(\%)]$ & $8(20.0)$ & $261(91.9)$ & $<0.001$ \\
\hline None & $32(80.0)$ & $23(8.1)$ & $<0.001$ \\
\hline One kind & $4(10.0)$ & $151(53.2)$ & \\
\hline Two kinds & $3(4.7)$ & $99(34.9)$ & \\
\hline Three or more kinds & $1(2.5)$ & $11(3.9)$ & \\
\hline Corticosteroids [n (\%)] & $1(2.5)$ & $50(17.6)$ & 0.014 \\
\hline Immunoglobulin [n (\%)] & $1(2.5)$ & 39 (13.7) & 0.043 \\
\hline Blood transfusion [n (\%)] & $1(2.5)$ & $4(1.4)$ & 1.000 \\
\hline \multicolumn{4}{|l|}{ Oxygen support [n (\%)] } \\
\hline None & $39(97.5)$ & $238(83.8)$ & 0.024 \\
\hline Nasal catheter, mask or other & $0(0.0)$ & $22(7.7)$ & \\
\hline Non-invasive ventilation & $0(0.0)$ & $8(2.8)$ & \\
\hline Invasive mechanical ventilation & $1(2.5)$ & $16(5.6)$ & \\
\hline Mortality [n (\%)] & $0(0.0)$ & $1(0.4)$ & 1.000 \\
\hline Required ICU support [n (\%)] & $1(2.5)$ & $32(11.3)$ & 0.047 \\
\hline Symptomatic duration $[\mathrm{d}$, median (IQR)] & $5(0-8)$ & $8(4-12)$ & $<0.001$ \\
\hline Hospital stay $[\mathrm{d} \text {, median }(\mathrm{IQR})]^{\mathrm{a}}$ & $15(10-23)$ & $6(5-8)$ & $<0.001$ \\
\hline
\end{tabular}

The duration of hospital stay was not used as a prognostic indicator in this study, because many children with COVID-19 were observed in the hospital after the symptoms completely disappeared for the possibility of infectivity 


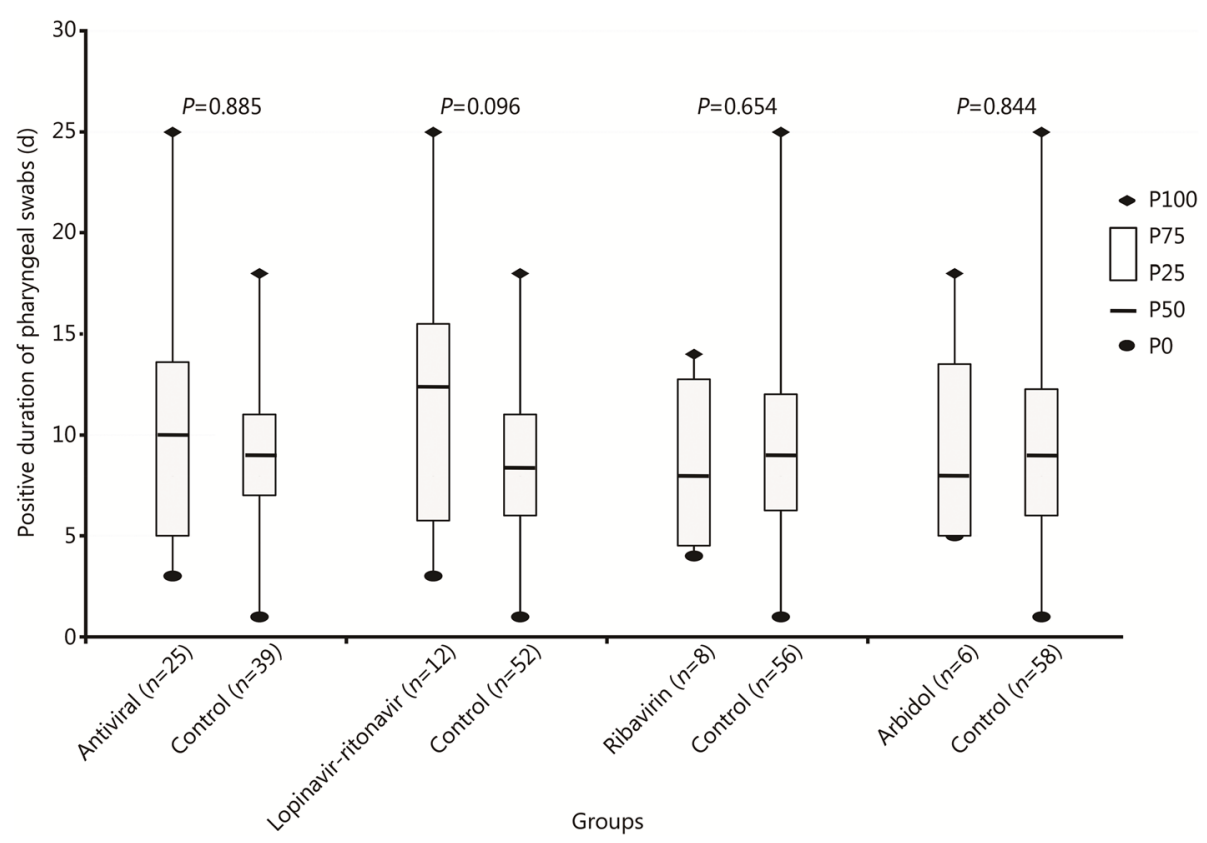

Fig. 2 Effects of lopinavir-ritonavir, ribavirin and arbidol on children with COVID-19. Lopinavir-ritonavir, ribavirin, and arbidol were used in about $1 / 3$ of children with COVID-19, but they did not shorten the duration of positive PCR results from pharyngeal swabs for children with COVID-19

indicators related to organ or tissue damage, D-dimer and secondary bacterial infection were also lower in the COVID-19 pneumonia. However, no significant differences were found between groups in the duration of positive PCR results from pharyngeal swabs after receiving antiviral treatment.

Respiratory illnesses are consistently troublesome for children in the winter months and early spring. SARS$\mathrm{CoV}-2$ is an emerging contagious pathogen causing a high prevalence of pneumonia. Understanding the clinical manifestations in children with COVID-19, especially those different from other similar diseases, is important for diagnosis, treatment and management of this disease. Clear exposure histories, GGO on CT images are the main characteristics of SARS-CoV-2 infection in children with COVID-19, and most children $(82.8 \%)$ in the present study cohort were from family cluster cases; however, family clusters of pneumonia were not common among other virus infections, because most adults already have immunity against other common respiratory viruses and will not develop pneumonia. GGO on CT images was a distinct characteristic of children with COVID-19, and did not occur in the other 284 viral pneumonia cases. However, the proportion of the occurrence of GGO was obviously lower than that of adult cases [14], and 55\% of COVID-19 pneumonia cases were without these typical radiographic changes. Moreover, it was observed in the present study that, after several days, GGO can transform into lesions that cannot differentiate COVID-19 from other lung infections, which makes it more difficult to identify children with COVID-19 by using CT images. Consolidation was more common in the CT images of other viral pneumonia cases, but this cannot be used to exclude COVID-19, as consolidation can be found in $12.5 \%$ of pediatric COVID-19 pneumonia cases. The epidemiology and the PCR tests are therefore essential in the diagnosis of COVID-19.

The prevalence of pneumonia in SARS-CoV-2 infection was $62.5 \%$, similar with that of SARS (65\%) [15], but much higher than that of H1N1 influenza (11\%) and many other viruses. No differences were reported in the proportion of children in different age groups who developed pneumonia after SARS-CoV-2 infection, and this situation can be observed in the research of Dong et al. [16]. and Wu et al. [6], indicating that younger age has no protective effect on preventing SARA-CoV-2 infection from developing COVID-19 pneumonia in children. Children in the present study were usually well cared for at home and had relatively fewer opportunities to be exposed to people with serious transmissible infection, which may help to explain the lower incidence of children with COVID-19 compared with adults.

The symptoms and disease severity of COVID-19 pneumonia in children were no more severe than other common viral pneumonia. Fever and cough were the most common symptoms of children with COVID-19 pneumonia. In the present study, the proportion of cases with fever among children with COVID-19 was significantly lower than that of adults with COVID-19 [13, 17], 
and that of children with other viral pneumonia. Fever temperatures were mainly low to moderate, and the proportion of cases with high fever was only $7.5 \%$, lower than that of other viral pneumonia cases (58.8\%); the duration of fever was also shorter in children with COVID-19 than those in other viral pneumonia. The proportion of severe cases (1/40) in children with COVID-19 pneumonia in the present study is consistent with previous results reported by Lu et al. [9]. (3 of 171 children with COVID-19) and Dong et al. [16]. (21 of 731 children with COVID-19), which were significantly lower than those of adult cases [18] and lower than pneumonia caused by RSV (19/133) and human adenovirus $(5 / 25)$. Also, the proportion of cases that required intensive care was lower and the symptomatic duration was short as compared with the viral pneumonia cohort. Therefore, we concluded that COVID-19 pneumonia in children was no more severe than pneumonia caused by common viruses from community exposure.

Uncontrolled inflammatory innate responses may lead to tissue and organ damage in SARS-CoV-2 infection [19]. High levels of cytokines were detected in adult patients with severe illness caused by SARS-CoV [20], MERS-CoV [21], and SARS-CoV-2 [18] infections. In addition, excessively increased inflammatory indicators (CRP, PCT) were observed in severe pneumonia cases caused by human adenovirus [22]. The only single case of critical COVID-19 among children in our study, had significantly elevated IL-6 (120.31 ng/L), IL-10 (33.38 ng/ $\mathrm{L})$, and PCT $(0.43 \mathrm{ng} / \mathrm{ml})$, and acute respiratory distress syndrome, acute renal failure and cardiac insufficiency occurred. However, compared to pneumonia caused by other viruses, we found that the inflammatory indicators (CRP, PCT, ESR, IL-6, LDH), as well as biochemical indicators associated with organ or tissue damage (ALT, AST, CK, CK-MB), and indexes associated with disseminated intravascular coagulation, were lower in the children with COVID-19 pneumonia. This indicates that SARS-CoV-2 infection has less effect on excessive activation of the innate immune system in children and rarely triggered cytokine storm, which may be associated with milder clinical manifestations of children with COVID-19. The only single severe case in the COVID19 pneumonia cohort was combined with hydronephrosis caused by kidney stones $(1 / 1$ vs. $1 / 39, P=0.037)$, and five severe cases in the other viral pneumonia cohort were found to have underlying chronic disease (5/38 vs. $15 / 246, P=0.112$ ). However, we cannot make any conclusion on the effects of comorbidities on progression to severe pneumonia.

Antiviral therapy, corticosteroids, immune therapy and antibiotics usage were the main components of COVID19 treatment. By activating innate immunity, interferon was nebulized in more than $90 \%$ of children in the
COVID-19 cohort in the present study as well as in those with other viral infections [23]. However, this study failed to evaluate the effects of interferon on SARS-CoV-2 infection. Lopinavir-ritonavir, ribavirin, and arbidol were used in about $1 / 3$ of children with COVID-19 in total, but they did not shorten the duration of positive PCR results from pharyngeal swabs for children with COVID-19. For the other viral pneumonia, oseltamivir and paramivir were used in influenza A and influenza B related pneumonia, but antiviral drugs were rarely used in pneumonia caused by other viruses. Since all 64 children with COVID-19 had a relatively good prognosis, we proposed that it was not necessary to aggressively administer antiviral drugs in children with COVID-19 before effective and safe antiviral drugs are confirmed. Nevertheless, several studies have shown that immune therapy may be beneficial in dealing with cytokine storm syndrome in adults with severe COVID-19 [24]. As mentioned above, cytokine storms were relatively rare and inflammatory indicators were lower in children with COVID-19 as compared to other viral pneumonia, so corticosteroids were not recommended as for other community-acquired viral pneumonia. However, corticosteroids might be tried for critical children with COVID-19 with obvious cytokine reactions, acute respiratory distress syndrome, multiple organ damage or with obvious wheezing. The percentage of viral-viral coinfection was $7.5 \%$ and viral-MP co-infection was $22.5 \%$ for children with COVID-19 pneumonia, which were similar between the viral pneumonia cohort and that reported by other studies on childhood viral pneumonia [25]. Secondary-infection with bacteria was detected in $18.5 \%$ cases of the viral pneumonia cohort, whereas none was found in the COVID-19 pneumonia cohort. Few cases were given penicillin for prophylactic treatment of possible bacterial infection. Milder symptoms and shorter symptomatic duration in children with COVID-19 may explain the low rate of secondary bacterial infections. For children with COVID-19, antibiotics are not used routinely, but multiple pathogen tests are recommended because of the potential of high proportions of co-infection in children with viral pneumonia.

The major limitation of our study was the selection bias. First, not all children with viral pneumonia were included since clinicians failed to identify causative agents in some individuals with pneumonia. Although all participating hospitals routinely tested pathogens in children with pneumonia, the relatively severe cases had more chances of pathogens being detected. Secondly, although the 64 SARS-CoV-2 infected patients contained cases from both Hubei province and outside Hubei province, their representativeness was limited due to a limited sample size. 


\section{Conclusions}

Younger age has no protective effect on preventing SARS-CoV-2 infection from developing into COVID-19 pneumonia in children. The symptoms and severity of children with COVID-19 pneumonia were no more severe than that of other viral pneumonia. Lopinavirritonavir, ribavirin, and arbidol do not shorten the duration of positive PCR results from pharyngeal swabs in children with COVID-19. Multi-respiratory-pathogen tests, including SARS-COV-2, are necessary and even during a COVID-19 outbreak, children with infection from other pathogens require sufficient attention.

\begin{abstract}
Abbreviations
APTT: Activated partial thromboplastin time; CAP: Community acquired pneumonia; CCDC: Chinese Center for Disease Control and Prevention; CK: Creatine kinase; CK-MB: Creatine kinase-MB; COVID-19: Coronavirus disease-2019; CRP: C-reactive protein; CT: Computed tomography; ESR: Erythrocyte sedimentation rate; GGO: Ground-glass opacity; IL6: Interleukin-6; LDH: Lactate dehydrogenase; MERS: Middle East respiratory syndrome; MP: Mycoplasma pneumonia; PCR: Polymerase chain-reaction; PCT: Procalcitonin; PT: Prothrombin time; RSV: Respiratory syncytial virus; SARS: Severe acute respiratory syndrome; WBC: White blood cell
\end{abstract}

\section{Acknowledgements}

We thank all the staffs who had contributed to these children's recovery. And thanks to unsigned contributors who cannot be cited here owing to significant space constraint.

\section{Authors' contributions}

GLR, GQX, LC, CZZ and YS did literature search and designed the study. GLR, $X F W$, JX, JL, QM, BH, WCZ, JL, CHT, SY, ZL, CX, FY, YZ, YZ, SLL, JFC, FY, YH, $\mathrm{BQW}$ and $Y S$ were responsible for disease diagnosis and treatment and data collection. GLR, GQX, JZ, ZT and MXM, YWW, CXL analysed data and wrote the report. LCH, CZZ and YS did data interpretation and revised the paper. All authors read and approved the final manuscript.

\section{Funding}

This work was supported by Scientific Research Project of Military Logistics Department, Grant Award Number: CLB20J032. The funders of this research had no role in study design, data collection and analysis, decision to publish, or preparation of the manuscript.

\section{Availability of data and materials}

The datasets used and/or analyzed during the present study are available from the corresponding author on reasonable request.

\section{Ethics approval and consent to participate}

The study protocol was reviewed and approved by the Medical Ethics Committee of General Hospital of Southern Theater Command of Chinese People's Liberation Army (approval number 2020-04). Signed informed consent of participants or their guardians was waived.

\section{Consent for publication}

Not applicable.

\section{Competing interests}

The authors declare that they have no competing interests.

\section{Author details}

${ }^{1}$ Department of Pediatrics, General Hospital of Southern Theater Command of PLA, 111 Liuhua Road, Yuexiu District, Guangzhou 510010, Guangdong, China. ${ }^{2}$ Department of Pediatrics, the Third People's Hospital of Shenzhen, Shenzhen 518100, Guangdong, China. ${ }^{3}$ Pediatric Intensive Care Unit, Wuhan Children's Hospital, Tongji Medical College, Huazhong University of Science \& Technology, Wuhan 430010, China. ${ }^{4}$ Pediatric Intensive Care Unit, Maternal and Child Health Hospital of Huangshi, Huangshi 435000, Hubei, China. ${ }^{5}$ Department of Pediatrics, the Second People's Hospital of Guangdong
Province, Guangzhou 510317, China. 'Department of Pediatrics, the Third Affiliated Hospital of Zunyi Medical University (the First People's Hospital of Zunyi), Guizhou 563000, China. ${ }^{7}$ Department of Pediatrics, the Eighth People's Hospital of Guangzhou, Guangzhou 510440, China. ${ }^{8}$ Department of Pediatrics, the First Affiliated Hospital of Xinxiang Medical University, Xinxiang 453100, Henan, China. 'Pediatric Intensive Care Unit, the Children's Hospital Zhejiang University School of Medicine, Hangzhou 310000, China.

${ }^{10}$ Department of Emergency / Critical Medicine, Children's Hospital of Nanjing Medical University, Nanjing 210008, China. ${ }^{11}$ Department of Pediatrics, the Second Affiliated Hospital of Guangzhou Medical University, Guangzhou 510260, China. ${ }^{12}$ Department of Medical information date room, General Hospital of Southern Theater Command of PLA, Guangzhou 510010, China. ${ }^{13}$ Department of Pediatrics, the First Affiliated Hospital of Jinan University, Guangzhou 510632, China. ${ }^{14}$ Department of Pediatrics, Maternal and Child Health Hospital of Yangjiang, Yangjiang 529500, Guangdong, China. ${ }^{15}$ Department of Pediatrics, Shenzhen Hospital Affiliated to the University of Chinese Academy of Sciences, Shenzhen 518107, Guangdong, China. ${ }^{16}$ Department of Pediatrics, Nanfang Hospital of Southern Medical University, Guangzhou 510515, China. ${ }^{17}$ Department of Pediatrics, Zhujiang Hospital of Southern Medical University, Guangzhou 510280, China.

${ }^{18}$ Department of Pediatrics, Military Hospital of 74 Group of PLA, Guangzhou 510318, China. ${ }^{19}$ Department of Neonatology, Children's Hospital of Chongqing Medical University/Ministry of Education Key Laboratory of Child/ Development and Disorders/National Clinical Research Center for Child Health and Disorders/Chongqing Key Laboratory of Pediatrics, Chongqing 400014, China. ${ }^{20}$ Department of Pediatrics, Zhongshan Boai Hospital, Zhongshan 528403, Guangdong, China. ${ }^{21}$ Department of Pediatrics, Strategic Support Force Medical Center of PLA, Beijing 100101, China.

Received: 15 July 2020 Accepted: 5 February 2021

Published online: 16 February 2021

\section{References}

1. Chen Y, Liu Q, Guo D. Emerging coronaviruses: genome structure, replication, and pathogenesis. J Med Virol. 2020;92(4):418-23.

2. Donnelly CA, Ghani AC, Leung GM, Hedley AJ, Fraser C, Riley S, et al. Epidemiological determinants of spread of causal agent of severe acute respiratory syndrome in Hong Kong. Lancet. 2003;361(9371):1761-6.

3. Cauchemez S, Fraser C, Van Kerkhove MD, Donnelly CA, Riley S, Rambaut A, et al. Middle East respiratory syndrome coronavirus: quantification of the extent of the epidemic, surveillance biases, and transmissibility. Lancet Infect Dis. 2014;14(1):50-6.

4. Li Q, Guan XH, Wu P, Wang XY, Zhou L, Tong YQ, et al. Early transmission dynamics in Wuhan, China, of novel coronavirus-infected pneumonia. $\mathrm{N}$ Engl J Med. 2020;382(13):1199-207.

5. Who. WHO coronavirus disease (COVID-19) dashboard. https://covid19.who. int/. Accessed 18 Jan 2021.

6. Wu ZY, Mcgoogan JM. Characteristics of and important lessons from the coronavirus disease 2019 (COVID-19) outbreak in China summary of a report of 72314 cases from the Chinese center for disease control and prevention. JAMA. 2020;323(13):1239-42.

7. Jiehao C, Jin X, Daojiong L, Zhi Y, Lei X, Zhenghai Q, et al. A case series of children with 2019 novel coronavirus infection: clinical and epidemiological features. Clin Infect Dis. 2020;71(6):1547-51. https://doi.org/10.1093/cid/ciaa198.

8. Qiu HY, Wu JH, Hong L, Luo YL, Song QF, Chen D. Clinical and epidemiological features of 36 children with coronavirus disease 2019 (COVID-19) in Zhejiang, China: an observational cohort study. Lancet Infect Dis. 2020;20(6):689-96.

9. Lu XX, Zhang LQ, Du H, Zhang JJ, Li YY, Qu JY, et al. SARS-CoV-2 infection in children. N Engl J Med. 2020;382(17):1663-5.

10. China National Health Commission. Diagnosis and treatment guidelines for 2019 novel coronavirus pneumonia (draft version 7). 2020; http://www.nhc. gov.cn/yzygj/s7653p/202003/46c9294a7dfe4cef80dc7f5912eb1989.shtml. Accessed 8 May 2020.

11. China National Health Commission. Guideline for diagnosis and treatment of community-acquired pneumonia in children (2019 version). Chin J Clin Infect Dis. 2019;12(12):6-13.

12. Stockmann C, Ampofo K, Killpack J, Williams DJ, Edwards KM, Grijalva CG, et al. Procalcitonin accurately identifies hospitalized children with low risk of bacterial community-acquired pneumonia. J Pediatric Infect Dis Soc. 2018: $7(1): 46-53$. 
13. Wang DW, Hu B, Hu C, Zhu FF, Liu X, Zhang J, et al. Clinical characteristics of 138 hospitalized patients with 2019 novel coronavirus-infected pneumonia in Wuhan, China. JAMA. 2020;323(11):1061-9.

14. Xu X, Yu CC, Qu J, Zhang LG, Jiang SF, Huang DY, et al. Imaging and clinical features of patients with 2019 novel coronavirus SARS-CoV-2. Eur J Nucl Med Mol Imaging. 2020:47(5):1275-80.

15. Babyn PS, Chu WCW, Tsou IYY, Wansaicheong GKL, Allen U, Bitnun A, et al. Severe acute respiratory syndrome (SARS): chest radiographic features in children. Pediatr Radiol. 2004;34(1):47-58.

16. Dong $Y$, Mo X, Hu Y, Qi X, Jiang F, Jiang Z, et al. Epidemiology of COVID-19 among children in China. Pediatrics. 2020;145(6):e20200702. https://doi.org/1 0.1542/peds.2020-0702.

17. Guan WJ, Ni ZY, Hu Y, Liang WH, Ou CQ, He JX, et al. Clinical characteristics of coronavirus disease 2019 in China. N Engl J Med. 2020;382(18):1708-20.

18. Huang CL, Wang YM, Li XW, Ren LL, Zhao JP, Hu Y, et al. Clinical features of patients infected with 2019 novel coronavirus in Wuhan, China. Lancet. 2020;395(10223):497-506.

19. Cao XT. COVID-19: immunopathology and its implications for therapy. Nat Rev Immunol. 2020;20(5):269-70.

20. Wong CK, Lam CWK, Wu AKL, Ip WK, Lee NLS, Chan IHS, et al. Plasma inflammatory cytokines and chemokines in severe acute respiratory syndrome. Clin Exp Immunol. 2004;136(1):95-103.

21. Falzarano D, De Wit E, Rasmussen AL, Feldmann F, Okumura A, Scott DP, et al. Treatment with interferon-alpha $2 b$ and ribavirin improves outcome in MERS-CoV-infected rhesus macaques. Nat Med. 2013;19(10):1313-7.

22. Shen CF, Wang SM, Ho TS, Liu CC. Clinical features of community acquired adenovirus pneumonia during the 2011 community outbreak in southern Taiwan: role of host immune response. BMC Infect Dis. 2017;17(1):196. https://doi.org/10.1186/s12879-017-2272-5.

23. Mesev EV, Ledesma RA, Ploss A. Decoding type I and III interferon signalling during viral infection. Nat Microbiol. 2019;4(6):914-24.

24. Mehta P, Mcauley DF, Brown M, Sanchez E, Tattersall RS, Manson JJ, et al. COVID-19: consider cytokine storm syndromes and immunosuppression. Lancet. 2020;395(10229):1033-4.

25. Haq IJ, Battersby AC, Eastham K, Mckean M. Community acquired pneumonia in children. BMJ. 2017;356:j686.

Ready to submit your research? Choose BMC and benefit from:

- fast, convenient online submission

- thorough peer review by experienced researchers in your field

- rapid publication on acceptance

- support for research data, including large and complex data types

- gold Open Access which fosters wider collaboration and increased citations

- maximum visibility for your research: over $100 \mathrm{M}$ website views per year

At $\mathrm{BMC}$, research is always in progress.

Learn more biomedcentral.com/submissions 